\title{
Large- and Small-Fiber Neuropathy in Patients with Tarlov Cysts
}

\author{
Mieke Hulens $\mathbb{D}^{\prime}$, Frans Bruyninckx $\mathbb{D}^{2}$, Dietmar Rudolf Thal $\mathbb{D}^{3,4}$, Ricky Rasschaert $\mathbb{D}^{5}$, \\ Chris Bervoets $\mathbb{D}^{6-8}$, Wim Dankaerts $\mathbb{D}^{1}$
}

'Department of Rehabilitation Sciences, Musculoskeletal Rehabilitation Research Unit, Faculty of Kinesiology and Rehabilitation Sciences, KU Leuven, Leuven, Belgium; ${ }^{2}$ Department of Physical Medicine \& Rehabilitation, EMG-Laboratory, University Hospitals of Leuven, Leuven, Belgium; ${ }^{3}$ Department of Imaging and Pathology, Laboratory of Neuropathology, and Leuven Brain Institute, KU Leuven, Leuven, Belgium; ${ }^{4}$ Department of Pathology, University Hospitals of Leuven, Leuven, Belgium; ${ }^{5}$ Department of Neurosurgery, AZ Rivierenland, Bornem, Belgium; ${ }^{6}$ Department of Neurosciences, Adult Psychiatry, KU Leuven, Leuven, Belgium; ${ }^{7}$ Department of Ophthalmology, University Hospitals of Leuven, Leuven, Belgium; ${ }^{8}$ Department Adult Psychiatry, University Psychiatric Center of KU Leuven, Leuven, Belgium

Correspondence: Mieke Hulens, Department of Rehabilitation Sciences, Musculoskeletal Rehabilitation Research Unit, Faculty of Kinesiology and Rehabilitation Sciences, University of Leuven, Tervuursevest I0I, Leuven, Belgium, Tel +32 478338003 , Fax +32 I6 32 9l 97, Email miekehulens@gmail.com

Purpose: Tarlov cysts (TCs) are dilated nerve root sheaths originating from increased cerebrospinal pressure. Patients with TCs often complain of neuropathic pain and paresthesia. The aim of this study was to retrospectively review intraepidermal nerve fiber density (IENFD) and electrodiagnostic (EDX) data from TC patients.

Patients and Methods: Lower leg skin biopsy results and EDX data from the L2-S4 myotomes of patients with lumbar or sacral TCs $\geq 8 \mathrm{~mm}$ were retrieved from a database of a physical medicine clinic. Patients with compressive pathology, diabetes mellitus and chemotherapy were excluded.

Results: IENFD data from 17 patients and EDX data from 24 patients with TCs $\geq 8 \mathrm{~mm}$ were available. The mean age was $47 \pm 10 \mathrm{y}$, and $83 \%$ were women. In $82 \%$ of patients, the IENFD was below the 5 th percentile by age and sex. EDX showed increased Hoffmann reflex latencies in $25 \%$, increased anal reflex latencies in $95 \%$, and a patchy distribution of neurogenic motor unit potentials in $100 \%$. More than $50 \%$ of needle EMG abnormalities appeared in myotomes unrelated to the location of the TCs.

Conclusion: Small- and/or large-fiber neuropathy was documented in a significant proportion of patients with TCs. The novel findings may add to the understanding of the mechanisms involved in symptomatic TCs. We propose that pathologically elevated cerebrospinal fluid pressure not only dilates some of the nerve root sheaths to form TCs but also potentially damages axons in nondilated nerve root sheaths and neurons in the dorsal root ganglia.

Keywords: perineural cysts, skin biopsies, radicular pain, anal reflex, nerve conduction studies, fibromyalgia

\section{Introduction}

Tarlov cysts (TCs) or perineural cysts are dilations of nerve root sheaths near the dorsal root ganglion containing nerve roots and neurons. The dilations are assumed to originate from moderately pathologically increased pulsatile cerebrospinal pressure forcing cerebrospinal fluid (CSF) into the nerve root sheaths. Nerve root dilations occur more frequently in the sacrum, where the hydrostatic pressure is the highest. ${ }^{1-3}$ The prevalence of TCs $\geq 5 \mathrm{~mm}$ on lumbar and sacral magnetic resonance imaging (MRI) performed for any reason is $13 \%{ }^{4}$ TCs produce symptoms more frequently than generally assumed. ${ }^{5}$ It has been estimated that approximately $25 \%$ of TCs are symptomatic and that an unknown percentage of asymptomatic TCs may become symptomatic later in life. ${ }^{6}$

Both large- and small-fiber neuropathy (SFN) have been documented in other chronic painful conditions, such as fibromyalgia ${ }^{7-9}$ and Ehlers-Danlos syndrome. ${ }^{10,11}$ Additionally, a high prevalence of TCs was found among patients with fibromyalgia $(39 \%)^{12}$ and there is some evidence of an association of TCs with connective tissue disorders, such as hypermobility-type Ehlers-Danlos syndrome and Marfan syndrome. ${ }^{13}$ 
Patients with TCs may show signs of motor nerve involvement, including radicular symptoms, sphincter dysfunction, or objective muscle weakness. ${ }^{14,15}$ Similar to patients with SFN, TC patients frequently complain of burning pain and paresthesia in the lower legs and feet. ${ }^{14}$ The latter symptoms could not always be linked to the presence of TCs and were suspected to be more likely related to small fiber neuropathy. Therefore, in the diagnostic workup, electrodiagnostic (EDX) tests or skin biopsies to assess intraepidermal nerve fiber density (IENFD) were performed.

The aim of this exploratory study was to retrospectively review IENFD analyses and EDX data assessing lumbar and sacral nerve root abnormalities in symptomatic TC patients.

\section{Materials and Methods}

The literature search for this manuscript used the MESH terms "Tarlov cysts", "small fiber neuropathy" and "electrodiagnosis" or "EMG". The literature was accessed using PubMed and Web of Science.

In this retrospective study, IENFD and EDX assessments of patients with chronic pain and with evidence of TCs on MRI were selected from the database of an outpatient physical medicine clinic from January 2016 to February 2021. The diameter of the TCs was measured on all available planes (sagittal, axial, and coronal planes), and the largest diameter of each TC was reported.

The inclusion criterion was the presence of lumbar or sacral cystic lesions $\geq 8 \mathrm{~mm}$. In the literature, there is no consensus regarding the size at which nerve root dilation is termed TC. One study reporting on the prevalence of TCs used $5 \mathrm{~mm}$ as the lower limit. ${ }^{4}$ However, most studies consider larger TCs. Therefore, we chose $8 \mathrm{~mm}$ as the lower limit for the inclusion of TCs in this study.

The exclusion criteria were compressive pathology, diabetes mellitus and past or current chemotherapy.

Skin biopsies were taken $10 \mathrm{~cm}$ above the malleolus externus using disposable circular 3-mm punch needles. Fixation and analysis were conducted in the Department of Pathology of UZ Leuven. Punch biopsies were fixed in $4 \%$ phosphate buffered formaldehyde for 6-24 hours and then incubated in a cryoprotective solution (10\% sucrose). Cross section of the epidermis was cut at a thickness of $50 \mu \mathrm{m}$ with a cryotome. Immunohistochemistry was carried out with a polyclonal rabbit antibody raised against Protein Gene Product 9.5 (1/100, DAKO, Glostrup, Denmark) using an automated immunostainer. It was first ensured that nerve fibers associated with glands and/or blood vessels were stained as internal controls. The number of nerve fibers that entered the epithelium was counted in three different cross-sections. ${ }^{16}$ The length of the epithelium was measured using ImageJ software (NIH, Bethesda, MD, USA).

EDX was performed by an expert electrophysiologist at UZ Leuven. A Natus Synergy electromyography machine (Natus Medical Incorporated, 6701 Koll Center Parkway Suite 120, Pleasanton, CA, USA) with filter settings of 20$20,000 \mathrm{~Hz}$ was used with Natus disposable concentric needle electrodes (30 G, 25-mm length).

IENFD was compared with sex- and age-specific normative values according to the Worldwide Reference Study of Lauria et al. ${ }^{17}$ Values $<0.05$ quantile for sex and age were considered a sign of SFN. Note that in this manuscript, the more common term of " 5 th percentile" will be used instead of the " 0.05 quantile" term used in Lauria et al's ${ }^{17}$ paper.

Nerve conduction studies included the sural nerves, fibular motor nerves, S1 Hoffmann reflexes and anal reflexes.

Needle EMG included the vastus medialis (L3), vastus lateralis (L4), extensor digitorum (L5), tibialis anterior (L4L5), gastrocnemius muscle medial head (S1), tibial nerve-supplied intrinsic foot muscles (S2), and external anal sphincter (S2, S3, S4).

During needle EMG, abnormal spontaneous activity at rest and the presence of $\geq 50 \%$ polyphasic motor unit potentials during contraction were considered pathological. ${ }^{18}$ Electrodiagnostic abnormality severity scores in each myotome were labeled as follows: $0=$ normal; $1=$ slight $(\geq 50 \%$ polyphasic MUPs); $2=$ moderate $(\geq 75 \%$ polyphasic MUPs); $3=$ severe ( $\geq 90 \%$ polyphasic MUPs).

For the anal reflex assessment, the EMG needle was inserted on the left and right sides of the anal sphincter at the mucocutaneous junction at a depth of $3 \mathrm{~mm}$. Electrical stimulation with a maximal pulse $(300 \mathrm{~V}, 35 \mathrm{~mA})$ and 1 -ms duration was applied caudally on the sacral midline. Latency was calculated from onset of the stimulation artifact to onset of the reflex response. ${ }^{19}$

Pearson correlation coefficients between the size of the TCs and the anal reflex parameters, and Spearman correlation coefficients between the size of the TCs and the severity of needle EMG abnormalities in the corresponding myotomes 
were calculated. For the calculation of the Pearson correlation coefficients between the size of the S2-S4 TCs and EMG abnormalities in the anal sphincter or the anal reflex latency, the largest TC of the S2, S3, or S4 nerve roots was considered because of their shared innervation of the anal sphincter.

Because of the retrospective nature of the study, the available MRIs were not intended for the visualization of sacral nerve roots. Thus, not all nerve roots were sufficiently visible to be measured. All nondilated lumbar and sacral nerve roots were included in the calculation using a uniform estimated mean diameter of $4 \mathrm{~mm}$.

Analyses were performed using SAS software (version 9.4, SAS System for Windows).

The study was conducted according to the guidelines of the Declaration of Helsinki and approved by the Ethics Committee Research of University Hospitals Leuven (protocol code S65053; date of approval: March 1, 2021). Patient consent was waived due to the retrospective nature of the analysis based on existing data. Patient data were accessed in compliance with relevant data protection and privacy regulations.

\section{Results}

In total, 31 patients with symptomatic TCs were included ( 26 women and 5 men; mean age 47/ 10 years; range $31-74$ years). EDX data from 24 patients (20 women and 4 men) and IENFD analyses from 17 patients (15 women and 2 men) were available. Eleven patients had undergone both IENFD and EDX studies.

The TCs were most frequently located on S2 nerve roots. The mean size of TCs showed a caudo-rostral gradient, with the largest TCs on S3 and S4 nerve roots and the smallest on L4 and L5 nerve roots. Table 1 displays the number and mean size ( \pm standard deviation) of the TCs.

Table 2 shows the results of IENFD analyses of the skin biopsy of 17 TC patients. IENFD was $<5$ th percentile for age and sex in $82 \%$ of patients. The remaining samples showed IENFD values slightly above the 5 th percentile.

Table 3 shows the EDX test results. Sural nerve latency was delayed $(>3.6 \mathrm{~ms})$ in one patient $(6 \%)$, and amplitude was low $(\leq 6 \mu \mathrm{V})$ in two patients $(13 \%)$. Hoffmann reflexes were delayed in $25 \%$ of TC patients, and anal reflexes were delayed in $95 \%$ ( $38 \%$ unilaterally; $57 \%$ bilaterally).

Twelve of 21 patients (57\%) with delayed anal reflex latency complained of mild (48\%) to severe (10\%) fecal incontinence.

Resting needle EMG showed fasciculation potentials in the S1 myotome in $14 \%$ and in the S2 myotome in $86 \%$ of patients. EMG during voluntary contraction showed patchy neurogenic motor unit potentials in the L2-S4 myotomes in all patients.

Table I Number and Size \pm Standard Deviation of TCs in 31 Patients on 62 (Left + Right) Nerve Roots from $L 2$ to $S 4$

\begin{tabular}{|l|c|c|c|}
\hline Nerve Roots & Number of TCs & Mean Size (mm) & Range (mm) \\
\hline L2 & 0 & & \\
\hline L3 & 0 & & $8-10$ \\
\hline L4 & 3 & $8.7 \pm 1.2$ & $8-10$ \\
\hline L5 & 11 & $8.5 \pm 0.8$ & $8-17$ \\
\hline S1 & 24 & $10.8 \pm 2.6$ & $8-34$ \\
\hline S2 & 28 & $12.6 \pm 6.2$ & $8-35$ \\
\hline S3 & 23 & $14.5 \pm 6.1$ & $10-20$ \\
\hline S4 & 6 & $14.3 \pm 3.4$ & \\
\hline
\end{tabular}

Abbreviation: TCs, Tarlov cysts. 
Table 2 Age, Results of IENFD Analyses, Mean Number of ESF/mm and 0.05 Quantile ESF/Mm Values for Age and Sex in Lower Leg Skin Biopsies of 17 Patients with TCs

\begin{tabular}{|l|l|l|l|l|l|l|l|l|l|l|l|l|l|l|l|l|l|l|}
\hline $\begin{array}{l}\text { Patient Code (Age in } \\
\text { Years) }\end{array}$ & A (61) & B (43) & C (38) & D (42) & E (4I) & F (49) & G (4I) & H (42) & I (61) & J (47) & K (54) & L (42) & M (42) & N (38) & O (43) & P (58) & Q (50) \\
\hline ESF/mm & $1.57^{*}$ & 5.71 & $5.24^{*}$ & $1.83^{*}$ & $5.37^{*}$ & $2.49^{*}$ & $0.71^{*}$ & $4.6^{*}$ & 3.35 & $1.05^{*}$ & 3.7 & $3.16^{*}$ & $2.72^{*}$ & $2.7^{*}$ & $1.88^{*}$ & $3.25^{*}$ & $1.65^{*}$ \\
\hline 5th percentile & 3.2 & 5.7 & 7.1 & 4.4 & 5.7 & 5.7 & 5.7 & 5.7 & 3.2 & 5.7 & 3.5 & 5.7 & 5.7 & 7.1 & 7.1 & 4.3 & 5.7 \\
\hline
\end{tabular}

Note: $*<0.05$ percentile.

Abbreviations: IENFD, intraepidermal nerve fiber density; ESF/mm, epidermal small fibers per millimeter; TCs, Tarlov cysts. 
Table 3 Results of Nerve Conduction Studies and Needle EMG in 23 Patients with TCs

\begin{tabular}{|c|c|c|c|}
\hline Nerves & \multicolumn{2}{|c|}{$\begin{array}{l}\text { Number of Patients with EDX } \\
\text { Abnormalities/Total Number of } \\
\text { Patients Examined and \% }\end{array}$} & \\
\hline \multicolumn{4}{|l|}{ Nerve conduction studies } \\
\hline \multicolumn{4}{|l|}{ Sural nerve } \\
\hline Latency $\geq 3.6 \mathrm{~ms}(14 \mathrm{~cm})$ & $1 / 16$ & $6 \%$ & \\
\hline Amplitude $\leq 6 \mu \mathrm{V}$ & $2 / 16$ & $13 \%$ & \\
\hline \multicolumn{4}{|l|}{ Peroneal nerve } \\
\hline Conduction velocity $>39 \mathrm{~m} / \mathrm{s}$ or latency $>6 \mathrm{~ms}$ & $0 / 19$ & $0 \%$ & \\
\hline Amplitude $<1000 \mu \mathrm{V}$ & $1 / 19$ & $5 \%$ & \\
\hline \multicolumn{4}{|l|}{ Hoffmann reflex } \\
\hline Latency $\geq 32 \mathrm{~ms}$; left/right difference $>2 \mathrm{~ms}$ & $5 / 20$ & $25 \%$ & \\
\hline \multicolumn{4}{|l|}{ Anal reflex } \\
\hline $\begin{array}{l}\text { Latency }>40 \mathrm{~ms} \text { (or }>45 \mathrm{~ms} \text { in women who have given birth vaginally); } \\
\text { left/right difference }>6 \mathrm{~ms}\end{array}$ & $20 / 21$ & $95 \%$ & \\
\hline Amplitude: left-right difference $\geq 500 \mu \mathrm{V}$ & $15 / 21$ & $71 \%$ & \\
\hline \multicolumn{3}{|l|}{ Needle EMG $\geq 50 \%$ neurogenic motor unit potentials } & $\begin{array}{c}\text { Mean severity score } \\
(0-3)\end{array}$ \\
\hline L2 (rectus femoris m.) & $1 / 18$ & $6 \%$ & \\
\hline L3 (vastus medialis m.) & $0 / 18$ & $0 \%$ & \\
\hline L4 (vastus lateralis m.) & $5 / 18$ & $29 \%$ & 0.3 \\
\hline L5 (extensor digitorum longus m.) & $13 / 18$ & $72 \%$ & 0.6 \\
\hline SI (medial head of gastrocnemius m.) & $4 / 20$ & $20 \%$ & 0.1 \\
\hline S2 (flexor hallucis brevis m.) & $18 / 22$ & $82 \%$ & 1.1 \\
\hline (S2) S3, S4 (external anal sphincter) & $18 / 22$ & $82 \%$ & 1.1 \\
\hline
\end{tabular}

Notes: Severity scores: $0=$ normal; $I$ = slight ( $\geq 50 \%$ polyphasic MUPs); 2 = moderate ( $\geq 75 \%$ polyphasic MUPs); $3=$ severe ( $\geq 90 \%$ polyphasic MUPs) abnormalities.

Abbreviations: ms, milliseconds; $\mu \mathrm{V}$, microvolts; m, meters; s, seconds; EMG, electromyography; MUPs, motor unit potentials.

There was no correlation between TC size and the severity of needle EMG abnormalities or between TC size and anal reflex latency or amplitude (Table 4).

Only in 43 myotomes did the needle EMG abnormalities correspond to the location of the TCs. Fifty-three myotomes showed EMG abnormalities without corresponding TCs, and 39 TCs were documented on nerve roots without EMG abnormalities in the corresponding myotomes.

\section{Discussion}

This retrospective study documents SFN in $82 \%$ and EDX abnormalities in $100 \%$ of patients with lumbar or sacral TCs measuring $\geq 8 \mathrm{~mm}$. To our knowledge, SFN in patients with TCs has not been reported in the literature. Furthermore, representative EDX data in this population are scarce.

\section{Tarlov Cysts}

TCs are dilated nerve root sheaths resulting from pathologically increased CSF pressure. Initially, a connection exists between the dilating nerve root sheath and the subarachnoid space of the spinal canal. In some cases, this connection may become narrowed. As a consequence, CSF is forced into the TCs; however, it is blocked from exiting the cyst (valve 
Table 4 Correlations Between TC Size and Severity of EDX Abnormalities and Between TC Size and Anal Reflex Parameters

\begin{tabular}{|c|c|c|c|c|c|c|}
\hline $\begin{array}{l}\text { Nerve } \\
\text { Roots }\end{array}$ & Needle EMG & $\begin{array}{l}\text { Spearman } \\
\text { Correlation }\end{array}$ & $\begin{array}{l}\text { 95\% Confidence } \\
\text { Interval }\end{array}$ & P value & N. Patients & N. Obs. \\
\hline L4 & Vastus lateralis muscle & 0.21 & $-0.13-0.50$ & 0.22 & 18 & 36 \\
\hline L5 & Extensor digitorum longus muscle & 0.08 & $-0.25-0.40$ & 0.63 & 18 & 36 \\
\hline SI & $\begin{array}{l}\text { Medial head of gastrocnemius } \\
\text { muscle }\end{array}$ & 0.32 & $-0.00-0.57$ & $0.05^{*}$ & 20 & 39 \\
\hline (S2), S3, S4 & External anal sphincter & 0.09 & $-0.22-0.38$ & 0.57 & 22 & 44 \\
\hline $\begin{array}{l}\text { Nerve } \\
\text { roots }\end{array}$ & Anal Reflex & Pearson correlation & $\begin{array}{l}\text { 95\% Confidence } \\
\text { interval }\end{array}$ & $P$ value & N. patients & N. obs. \\
\hline$(\mathrm{S} 2), \mathrm{S3}, \mathrm{S} 4$ & Anal reflex latency & 0.33 & $0.02-0.57$ & $0.03 *$ & 21 & 42 \\
\hline$(\mathrm{S} 2), \mathrm{S3}, \mathrm{S} 4$ & Anal reflex amplitude & 0.25 & $-0.52-0.07$ & 0.12 & 20 & 40 \\
\hline
\end{tabular}

Note: *Statistically significant.

Abbreviations: N. patients, number of patients; N. obs., number of observations.

mechanism). As a result, the rising pressure within the TC may compress the nerve root fibers inside, causing radicular pain in the corresponding dermatome.

In general, large TCs $(>20-30 \mathrm{~mm})$ are more often valved cysts because the pressure inside is higher than that in nonvalved cysts, causing them to grow faster. These valved TCs are rare; however, they are typically reported in the literature because the link between such TCs and symptoms is apparent. ${ }^{3,15,20}$ However, small TCs and TCs open to the spinal subarachnoid space (nonvalved cysts) may cause significant pain, as has been observed previously, ${ }^{3,20,21}$ although the reason is currently unclear.

Using conventional MRI images, our study was unable to differentiate between valved and nonvalved TCs. For this differentiation, CT or MRI myelography with intrathecal contrast injection is needed. Late filling (after several hours) of a TC indicates a valve mechanism. ${ }^{3}$ These imaging procedures were not implemented in our study due to the invasive nature of intrathecal contrast injection.

\section{Small-Fiber Neuropathy}

SFN is the consequence of damage to small-diameter thinly myelinated A $\delta$ - and unmyelinated C-fibers. Small fibers are responsible for nociceptive processing, thermal sensation, and autonomic function. ${ }^{22}$ SFN may produce debilitating sensory symptoms, including burning pain, hyperesthesia, and dysesthesia. ${ }^{23}$ Approximately half of SFN cases are idiopathic. $^{22,24}$

The assessment of IENFD at the lower limb by the immunohistochemical analysis of skin biopsy samples has become the gold standard to confirm damage to the small nerve fibers. ${ }^{23}$

In our cohort of patients with TCs and chronic pain, a significant percentage had IENFD below the 5th percentile for the diagnosis of SFN, whereas the remaining percentages were at or only slightly above the 5 th percentile for their age and sex.

Visceral sensory and autonomic neurons contribute to visceral reflex arcs, such as defecation and micturition. Thus, injury to small visceral fibers may explain why patients with TCs often suffer bowel, bladder and sphincter dysfunction. Based on our findings, other characteristic TC symptoms may be related to SFN, including perineal pain, leg weakness, restless legs, paresthesia, neuropathic pain, bladder pain, sexual dysfunction, chronic prostatitis, chronic pelvic pain and vulvodynia. $5,22,25$ 


\section{Large-Fiber Neuropathy}

The sural nerve contains fibers of nerve roots S1 and S2. Despite the fact that TCs were prevalent on S2, we documented delayed sural nerve response latency in only $6 \%$ and low sural nerve amplitudes in only $13 \%$ of patients. It is possible that in most patients, sural nerve conduction and amplitude were normal because the S1 nerve fibers were not affected.

An EDX study by Cattaneo et $\mathrm{al}^{26}$ in $11 \mathrm{TC}$ patients documented more prevalent sural nerve abnormalities (50\%). The study also revealed a correlation between the side of sural nerve abnormalities and the location of the TCs. However, no correlation between TC size and the severity of EDX abnormalities was observed. ${ }^{26}$ The reason why sural nerve abnormalities were more prevalent in the latter study than in ours may be due to the larger size of the TCs in that study. In Cattaneo et al's ${ }^{26}$ study, the TC size ranged from 20 to $30 \mathrm{~mm}$. As mentioned previously, large TCs more frequently tend to be valved cysts, in which the pressure inside rises to high levels and, therefore, creates greater compression of the nerve root inside. Due to their size, large TCs may also compress neighboring nerve roots, such as nerve root $\mathrm{S} 1$.

In our study, only 1 patient with a large TC $(34 \mathrm{~mm})$ underwent sural nerve response testing. This patient indeed showed a delayed sural nerve response on the same side as the large TC.

Our study revealed normal motor fibular nerve conduction in $95 \%$ of patients. This is in agreement with two studies conducted in $11 \mathrm{TC}$ patients ${ }^{26}$ and in $32 \mathrm{TC}$ patients. ${ }^{27}$ Motor nerve conduction was within normal limits in all patients.

We documented delayed S1 Hoffmann reflexes in $25 \%$ of patients (6 patients). Only two of these 6 patients showed needle EMG abnormalities in the corresponding S1 muscles, possibly indicating sensory S1 radiculopathy. In the literature, only one publication on a delayed Hoffmann reflex with associated needle EMG abnormalities in one TC patient was available. ${ }^{28}$

The (S2)-S3-S4 anal reflex arc consists of a sensory limb and a motor limb. Assessing the anal reflex thus has the advantage of including the sensory nerve, whereas needle EMG evaluates only motor innervation. ${ }^{29,30}$ Moreover, for electrophysiologists, the anal reflex is easier to measure and interpret than needle EMG of the external anal sphincter.

In our study, $95 \%$ of patients showed significantly increased anal reflex latency. No prior anal reflex studies in TC patients are available in the literature.

A delayed anal reflex affects bowel continence. In our study, 57\% of patients with unilaterally or bilaterally delayed anal reflex latency complained of mild to severe fecal incontinence. This is in accordance with other studies, in which clinically, mild to severe fecal incontinence was reported in 55 to $65 \%$ of patients with TCs. ${ }^{14,15}$

From these retrospective findings of anal reflex abnormalities in such a high percentage of TC patients, we propose that EDX anal reflex testing may be an additional objective tool for the diagnosis of symptomatic TC disease.

We found a diffuse, patchy distribution of needle EMG abnormalities in the lumbar or sacral nerve roots in all patients with TCs.

Additionally, we found only a weak significant positive correlation between S1 TC sizes and the severity of needle EMG abnormalities $(0.32 ; \mathrm{p}=0.05)$, and only a weak significant positive correlation between $\mathrm{S} 2, \mathrm{~S} 3$, S4 TCs sizes and anal reflex latency $(0.33 ; \mathrm{p}=0.03)$. This finding is in agreement with previous findings on this discordant relationship. ${ }^{26,31}$ Naderi $^{31}$ asserted that fluctuations in pain severity and lack of correlation between radiological findings and symptomatology are the main characteristics of TCs. Lim et $\mathrm{al}^{32}$ found a discordant relationship between TCs and pudendal neuralgia. In a meta-analysis of 22 radiological studies, Klepinowski et $\mathrm{al}^{33}$ found a correspondence with symptoms in only $16 \%$ of TCs.

Clinically, small TCs are assumed to be harmless, while large TCs are able to compress neighboring nerve roots (external compression). However, a TC is a dilated nerve root sheath. Therefore, compression of nerve root fibers and neurons occurs inside the cyst (internal compression).

We propose that the increased pulsatile spinal CSF pressure forcefully dilates some of the nerve root sheaths to initiate TC formation. Indeed, it has been documented that patients with idiopathic intracranial hypertension often have radicular symptoms and that nerve roots may be significantly dilated. Moreover, in patients with intracranial hypertension, paresthesia, and lower back pain may resolve following lumbar puncture with spinal fluid evacuation. ${ }^{34-39}$ At the same time, the increased spinal CSF pressure may also compress or damage axons and neurons inside both dilated and nondilated nerve root sheaths. 
These effects of moderately pathologically increased CSF pressure in patients with TCs can be compared with the effects of moderately increased blood pressure on the blood vessels. Moderate arterial hypertension may dilate some but not all arterial blood vessels, resulting in the formation of aneurysms. ${ }^{40}$ At the same time, moderate arterial hypertension progressively damages the inner layer of both dilated and nondilated blood vessels to cause atherosclerosis.

Damage to axons and neurons due to increased CSF pressure in both dilated and nondilated nerve root sheaths may explain the discordant relationship between TC location or size and EDX abnormalities or pain.

\section{Weaknesses of the Study}

The main limitation of this study is the lack of data from healthy control subjects. Therefore, we compared IENFD with normative values from the Worldwide Reference Study, ${ }^{17}$ and we compared EDX results with generally accepted normative values in the literature. ${ }^{18,19}$

Additionally, because this was a retrospective study, some data were missing, such as pain scores or the side on which the skin biopsy was taken. Thus, we were unable to correlate these data with TC size or location.

This exploratory study involved a relatively small sample of TC patients. Interpretation of such information can be biased, and the results cannot be accurately extrapolated to the general population.

\section{Conclusions}

This retrospective study documented signs of SFN in most patients and large fiber neuropathy in all patients with TCs $\geq$ $8 \mathrm{~mm}$. This is the first study to report SFN in patients with TCs, and ours is the largest TC cohort in which both nerve conduction and needle EMG data have been reported.

From our findings, we propose that IENFD measurement in lower leg skin biopsies and EDX of lumbar and sacral nerve roots (in particular anal reflex latency) can be considered reliable diagnostic tools for symptomatic TCs.

We propose that the same moderately increased pulsatile CSF pressure that initiates TC formation also damages axons and neurons inside nerve root sheaths and dorsal root ganglia.

\section{Abbreviations}

TCs, Tarlov cysts; IENFD, intraepidermal nerve fiber density; EDX, electrodiagnosis; CSF, cerebrospinal fluid; MRI, magnetic resonance imaging; SFN, small fiber neuropathy.

\section{Data Sharing Statement}

The dataset was submitted as Supplemental Material.

\section{Ethics Approval and Informed Consent}

The study was conducted according to the guidelines of the Declaration of Helsinki and approved by the Ethics Committee Research of University Hospitals Leuven (protocol code S65053; date of approval: March 1, 2021). Patient consent was waived due to the retrospective nature of the analysis based on existing data. Patient data were accessed in compliance with relevant data protection and privacy regulations.

\section{Funding}

This research received no external funding.

\section{Disclosure}

Dietmar Rudolf Thal has received speaker honoraria from Novartis Pharma Basel (Switzerland) and Biogen (USA); received travel reimbursement from GE Healthcare (UK) and UCB (Belgium); and collaborated with GE Healthcare (UK), Novartis Pharma Basel (Switzerland), Probiodrug (Germany), and Janssen Pharmaceutical Companies (Belgium). $\mathrm{He}$ is currently funded by Fonds Wetenschappelijk Onderzoek and SAO-FRA (Belgium). The authors report no other conflicts of interest associated with this work. 


\section{References}

1. Paulsen RD, Call GA, Murtagh FR. Prevalence and percutaneous drainage of cysts of the sacral nerve root sheath (Tarlov cysts). AJNR Am J Neuroradiol. 1994;15(2):293-297; discussion 298-299.

2. Shams PN, Goadsby PJ, Crockard HA, Casey AT, Plant GT. Paroxysmal raised intracranial pressure associated with spinal meningeal cysts. J Neurol. 2005;252(3):273-282. doi:10.1007/s00415-005-0430-y

3. Feigenbaum F, Henderson F. Tarlov cysts. In: Benzel E, editor. Spine Surgery. Philadelphia: Elsevier; 2012:1135-1140.

4. Kuhn FP, Hammoud S, Lefevre-Colau MM, Poiraudeau S, Feydy A. Prevalence of simple and complex sacral perineural Tarlov cysts in a French cohort of adults and children. $J$ Neuroradiol. 2017;44(1):38-43. doi:10.1016/j.neurad.2016.09.006

5. Hulens M, Rasschaert R, Bruyninckx F, et al. Symptomatic Tarlov cysts are often overlooked: ten reasons why-a narrative review. Eur Spine J. 2019;28(10):2237-2248. doi:10.1007/s00586-019-05996-1

6. Oaklander AL. Tarlov cysts. Neurosurg Focus. 2012;32(4):E9; author reply E9. doi:10.3171/2012.1.FOCUS11374

7. Martinez-Lavin M. Fibromyalgia and small fiber neuropathy: the plot thickens Clin Rheumatol. 2018;37(12):3167-3171. doi:10.1007/s10067-018$4300-2$

8. Caro XJ, Galbraith RG, Winter EF. Evidence of peripheral large nerve involvement in fibromyalgia: a retrospective review of EMG and nerve conduction findings in 55 FM subjects. Eur J Rheumatol. 2018;5(2):104-110. doi:10.5152/eurjrheum.2018.17109

9. Hulens M, Bruyninckx F, Rasschaert R, et al. Electrodiagnostic abnormalities associated with fibromyalgia. J Pain Res. 2020;13:737-744. doi:10.2147/JPR.S234475

10. Voermans NC, van Alfen N, Pillen S, et al. Neuromuscular involvement in various types of Ehlers-Danlos syndrome. Ann Neurol. 2009;65 (6):687-697. doi:10.1002/ana.21643

11. Cazzato D, Castori M, Lombardi R, et al. Small fiber neuropathy is a common feature of Ehlers-Danlos syndromes. Neurology. 2016;87 (2):155-159. doi:10.1212/WNL.0000000000002847

12. Hulens M, Bruyninckx F, Dankaerts W, et al. High prevalence of perineural cysts in patients with fibromyalgia and chronic fatigue syndrome. Pain Med. 2021;22(4):883-890. doi:10.1093/pm/pnaa410

13. Henderson FC, Austin C, Benzel E, et al. Neurological and spinal manifestations of the Ehlers-Danlos syndromes. Am J Med Genet C Semin Med Genet. 2017;175(1):195-211. doi:10.1002/ajmg.c.31549

14. Hulens MA, Dankaerts W, Rasschaert R, et al. Can patients with symptomatic Tarlov cysts be differentiated from patients with specific low back pain based on comprehensive history taking? Acta Neurochir. 2018;160(4):839-844. doi:10.1007/s00701-018-3494-z

15. Murphy K, Oaklander AL, Elias G, Kathuria S, Long DM. Treatment of 213 patients with symptomatic Tarlov cysts by CT-guided percutaneous injection of fibrin sealant. AJNR Am J Neuroradiol. 2016;37(2):373-379. doi:10.3174/ajnr.A4517

16. Lauria G, Cornblath DR, Johansson O, et al. EFNS guidelines on the use of skin biopsy in the diagnosis of peripheral neuropathy. Eur J Neurol. 2005;12(10):747-758. doi:10.1111/j.1468-1331.2005.01260.x

17. Lauria G, Bakkers M, Schmitz C, et al. Intraepidermal nerve fiber density at the distal leg: a worldwide normative reference study. J Peripher Nerv Syst. 2010;15(3):202-207. doi:10.1111/j.1529-8027.2010.00271.x

18. Preston DC, Shapiro BE. Needle electromyography. Fundamentals, normal and abnormal patterns. Neurol Clin. 2002;20(2):361-396. doi:10.1016/ S0733-8619(01)00005-6

19. Vodusek DB, Janko M, Lokar J. Direct and reflex responses in perineal muscles on electrical stimulation. J Neurol Neurosurg Psychiatry. 1983;46 (1):67-71. doi:10.1136/jnnp.46.1.67

20. Langdown AJ, Grundy JR, Birch NC. The clinical relevance of Tarlov cysts. J Spinal Disord Tech. 2005;18(1):29-33. doi:10.1097/01. bsd.0000133495.78245.71

21. Sun J, Wang Z, Li Z, et al. Reconstruction of nerve root sheaths for sacral extradural spinal meningeal cysts with spinal nerve root fibers. Sci China Life Sci. 2013;56(11):1007-1013. doi:10.1007/s11427-013-4536-7

22. Tseng MT, Pan CL, Hsieh ST. Overview of small fiber neuropathy. In: Hsieh ST, Anand P, Gibbons CH, Sommer C, editors. Small Fiber Neuropathy and Related Syndromes: Pain and Neurodegeneration. Singapore: Springer Nature; 2019:3-10.

23. Provitera V, Gibbons CH, Wendelschafer-Crabb G, et al. The role of skin biopsy in differentiating small-fiber neuropathy from ganglionopathy. Eur J Neurol. 2018;25(6):848-853. doi:10.1111/ene.13608

24. Oaklander AL, Nolano M. Scientific advances in and clinical approaches to small-fiber polyneuropathy: a review. JAMA Neurol. 2019;76 (10):1240-1251. doi:10.1001/jamaneurol.2019.2917

25. Privitera R, Praveen A. Visceral pain and hypersensitivity disorders. In: Hsieh ST, Anand P, Gibbons CH, Sommer C, editors. Small Fiber Neuropathy and Related Syndromes: Pain and Neurodegeneration. Singapore: Springer; 2019:3-179.

26. Cattaneo L, Pavesi G, Mancia D. Sural nerve abnormalities in sacral perineural (Tarlov) cysts. J Neurol. 2001;248(7):623-624. doi:10.1007/ s004150170144

27. Marino D, Carluccio MA, Di Donato I, et al. Tarlov cysts: clinical evaluation of an Italian cohort of patients. Neurol Sci. 2013;34(9):1679-1682. doi:10.1007/s10072-013-1321-0

28. Nadler SF, Bartoli LM, Stitik TP, Chen B. Tarlov cyst as a rare cause of S1 radiculopathy: a case report. Arch Phys Med Rehabil. 2001;82 (5):689-690. doi:10.1053/apmr.2001.22353

29. Pedersen E, Harving H, Klemar B, Torring J. Human anal reflexes. J Neurol Neurosurg Psychiatry. 1978;41(9):813-818. doi:10.1136/jnnp.41.9.813

30. Broens PM, Penninckx FM, Ochoa JB. Fecal continence revisited: the anal external sphincter continence reflex. Dis Colon Rectum. 2013;56 (11):1273-1281. doi:10.1097/DCR.0b013e3182a42d16

31. Naderi S. Surgical approaches in symptomatic Tarlov cysts. World Neurosurg. 2016;86:20-21. doi:10.1016/j.wneu.2015.10.044

32. Lim VM, Khanna R, Kalinkin O, Castellanos ME, Hibner M. Evaluating the discordant relationship between Tarlov cysts and symptoms of pudendal neuralgia. Am J Obstet Gynecol. 2020;222(1):70.e1-70.e6. doi:10.1016/j.ajog.2019.07.021

33. Klepinowski T, Orbik W, Sagan L. Global incidence of spinal perineural Tarlov's cysts and their morphological characteristics: a meta-analysis of 13,266 subjects. Surg Radiol Anat. 2021;43(6):855-863. doi:10.1007/s00276-020-02644-y

34. Bortoluzzi M, Di Lauro L, Marini G. Benign intracranial hypertension with spinal and radicular pain. Case report. J Neurosurg. 1982;57 (6):833-836. doi:10.3171/jns.1982.57.6.0833 
35. Round R, Keane JR. The minor symptoms of increased intracranial pressure: 101 patients with benign intracranial hypertension. Neurology. 1988;38(9):1461-1464. doi:10.1212/WNL.38.9.1461

36. Moosa A, Joy MA, Kumar A. Extensive radiculopathy: another false localising sign in intracranial hypertension. J Neurol Neurosurg Psychiatry. 2004;75(7):1080-1081.

37. Groves MD, McCutcheon IE, Ginsberg LE, Kyritsis AP. Radicular pain can be a symptom of elevated intracranial pressure. Neurology. 1999;52 (5):1093-1095. doi:10.1212/WNL.52.5.1093

38. Kincaid O, Rowin J. Intracranial hypertension causing polyradiculopathy and late or absent F-waves. J Neurol Neurosurg Psychiatry. 2006;77 (12):1384-1386. doi:10.1136/jnnp.2006.092387

39. Wall M, Kupersmith MJ, Kieburtz KD, et al. The idiopathic intracranial hypertension treatment trial: clinical profile at baseline. JAMA Neurol. 2014;71(6):693-701. doi:10.1001/jamaneurol.2014.133

40. Sun JJ, Wang ZY, Teo M, et al. Comparative outcomes of the two types of sacral extradural spinal meningeal cysts using different operation methods: a prospective clinical study. PLoS One. 2013;8(12):e83964. doi:10.1371/journal.pone.0083964

Journal of Pain Research

Dovepress

\section{Publish your work in this journal}

The Journal of Pain Research is an international, peer reviewed, open access, online journal that welcomes laboratory and clinical findings in the fields of pain research and the prevention and management of pain. Original research, reviews, symposium reports, hypothesis formation and commentaries are all considered for publication. The manuscript management system is completely online and includes a very quick and fair peer-review system, which is all easy to use. Visit http://www.dovepress.com/testimonials.php to read real quotes from published authors.

Submit your manuscript here: https://www.dovepress.com/journal-of-pain-research-journal 